\title{
Correlação entre a deflexão intrinsecoide, classificação clínica e parâmetros ecocardiográficos em cães com degeneração mixomatosa valvar mitral
}

[Correlation between intrinsecoid deflection, clinical classification and echocardiographic parameters in dogs with degenerative mitral valve myxomatosis]

\author{
D.C. Veiga ${ }^{1}$, G.C. Santos ${ }^{2}$, M.G. Sousa ${ }^{3}$
}

${ }^{1}$ Médica Veterinária Autônoma - São Paulo, SP

${ }^{2}$ Engenheiro de Bioprocessos e Biotecnologia Autônomo - São Paulo, SP

${ }^{3}$ Universidade Federal do Paraná - Curitiba, PR

\begin{abstract}
RESUMO
A degeneração mixomatosa valvar mitral (DMVM) costuma ser diagnosticada pelo ecocardiograma, porém o eletrocardiograma (ECG) pode sugerir alterações específicas e auxiliar no diagnóstico e no tratamento. A deflexão intrinsecóide (DI) é uma medida simples do ECG, que representa o início da despolarização ventricular e pode indicar a presença de sobrecargas e hipertrofía no ventrículo esquerdo. O objetivo deste trabalho é comparar dados prévios sobre condição clínica e ecocardiograma de cães com endocardiose de mitral e o valor da deflexão intrinsecóide do ECG, para buscar uma relação de concordância. Foram selecionados os arquivos de 45 cães anteriormente atendidos na rotina clínica do HV-UPFR. A estatística mostrou diferença relevante nos grupos B1, B2 e C nas derivações D2, D3, aVF, rV, V2 e V4 ( $<<0,05)$, o que permitiu classificá-los de acordo com o tamanho da DI, e esse resultado correspondeu à classificação do consenso de endocardiose de mitral em cães nesses estágios. Concluiu-se que há um aumento gradativo no valor da DI à medida que a DMVM avança, principalmente nos estágios B1, B2 e C, associado à sobrecarga e à hipertrofia ventricular esquerda, portanto esse parâmetro pode ser usado na classificação da doença.
\end{abstract}

Palavras-chave: eletrocardiograma, endocardiose de mitral, hipertrofia ventricular, insuficiência cardíaca, cardiologia

\begin{abstract}
The mitral valve myxomatous degeneration (DMVM) is usually diagnosed by echocardiography, however, electrocardiogram (ECG) may suggest specific alterations and aid diagnosis and treatment. Intrinsicoid deflection (DI) is a simple ECG measure that represents the onset of ventricular depolarization and may indicate the presence of overload and hypertrophy in the left ventricle. The objective of this study is to compare previous data on clinical condition and echocardiogram of dogs with mitral endocardiosis and the value of the intrinsicoid deflection from ECG to obtain a concordance relation. The archives of 45 previously examined dogs in the clinical routine of $H V-U F P R$ were selected. The statistic showed a significant difference in groups B1, B2 and C in leads D2, D3, aVF, rV, V2 and V4 $(P<0,05)$, which allowed to classify them according to the DI size, and this result corresponded to the consensus classification of mitral endocardiosis in dogs at these stages. It was concluded that there is a gradual increase in the value of DI as DMVM progresses, especially in stages B1, B2 and C, associated with left ventricular overload and hypertrophy, so this parameter can be used to classify the disease.
\end{abstract}

Keywords: electrocardiogram, mitral endocardiosis, ventricular hypertrophy, heart failure, cardiology

Recebido em 23 de janeiro de 2018

Aceito em 25 de janeiro de 2019

E-mail: veiga.dany@gmail.com 


\section{INTRODUÇÃO}

Degeneração mixomatosa valvar mitral (DMVM) é a doença cardíaca mais comum em cães e a causa mais frequente de insuficiência cardíaca congestiva nessa espécie; a prevalência varia com a idade e, especialmente em cães idosos de raças pequenas, aproxima-se de 100\% (Borgarelli e Buchanan, 2012). É uma condição particularmente evidente em raças de porte pequeno e, entre os mecanismos propostos para explicar essa predisposição, estão a proporção do coração em relação ao corpo, o tempo de vida mais longo e fatores genéticos envolvendo genes responsáveis pelo tamanho diminuto e o desenvolvimento da doença (Parker e KilroyGlynn, 2012).

O exame padrão-ouro para diagnóstico da doença valvar atualmente é o ecocardiograma transtorácico, pois permite, de modo não invasivo, detectar precocemente lesões valvares, inclusive em cães assintomáticos, e avaliar a regurgitação mitral, o remodelamento cardíaco, a função miocárdica e alterações no lado esquerdo do coração (Chetboul e Tissier, 2012). A técnica, no entanto, apresenta desvantagens, como a necessidade de operadores treinados, o alto custo e o maior tempo de execução, o que inviabiliza seu uso em larga escala (Häggstrom et al., 2004).

O eletrocardiograma (ECG) é um exame simples, de baixo custo, rápido, acessível e não operadordependente. Permite identificar sobrecargas nas câmaras cardíacas e prolongamento nos tempos de despolarização e repolarização, os quais podem auxiliar no diagnóstico da DMVM, além da função primordial de diagnosticar arritmias cardíacas. O traçado nesses pacientes geralmente é normal, podendo haver indícios de dilatação atrial e ventricular esquerdas, pelo aumento da largura da onda $\mathrm{P}$ e do complexo QRS e da amplitude da onda $\mathrm{R}$, e presença de arritmias, especialmente taquicardias sinusal e supraventricular e fibrilação atrial, ainda que a arritmia sinusal seja a mais comum (Tilley, 1992; Petrus e Larsson, 2015).

No estudo conduzido por Crosara et al. (2010), foi encontrado um percentual alto de cães com DMVM apresentando distúrbios de ritmo supraventricular e ventricular; as arritmias ventriculares pareceram mais comuns no grupo que apresentou mais sinais clínicos. Outro estudo semelhante, avaliando arritmias e parâmetros do Holter em Cavalier King Charles Spaniel portadores de endocardiose de mitral, não encontrou associação entre a doença e arritmias supraventriculares, apenas alterações como arritmia sinusal, taquicardia sinusal e bradicardia, associadas à gravidade da endocardiose e também à idade dos animais (Rasmussen et al., 2012). Ao avaliarem alterações de ritmo em cardiopatas com DMVM em estágios mais avançados (insuficiência cardíaca) por meio de ECG e Holter, Oliveira et al. (2014) detectaram taquicardia sinusal em quase todos os cães e uma alta incidência de arritmias supraventriculares naqueles cujo ecocardiograma revelou aumento de átrio, porém não foram detectadas arritmias ventriculares por nenhuma das modalidades diagnósticas.

Como esses sinais eletrocardiográficos nem sempre estão presentes nos pacientes acometidos pela doença valvar, o requerimento do ECG muitas vezes é desestimulado, sendo solicitados outros exames, como o ecocardiograma e a radiografia torácica. Porém, vários estudos em medicina já demonstraram que o ECG pode trazer informações valiosas quanto ao prognóstico de cardiopatas, especialmente em relação à sobrecarga de volume e à hipertrofia ventricular esquerda.

Um sistema utilizado na medicina para diagnóstico dessa condição em humanos considera diversas alterações ao longo do traçado e para cada uma delas concede um ponto; entre elas, estão a duração do QRS (igual ou superior a $0,09 \mathrm{~s}$ ) e da deflexão intrinsecóide (DI) (igual ou superior a $0,05 \mathrm{~s}$ em V5 ou V6), definida como o tempo desde o início do QRS até o pico da onda R (Romhilt e Estes, 1968). Representa o início da despolarização ventricular a partir da superfície endocárdica e epicárdica ventricular esquerda, sendo uma medida mais precisa da ativação elétrica do VE do que a duração do QRS, que mensura a despolarização ventricular completa (O’neal et al., 2016).

Na revisão de literatura conduzida por PérezRiera et al. (2016), os pesquisadores propuseram listar as aplicações clínicas desse parâmetro do ECG, sendo os principais: sobrecarga sistólica e diastólica do ventrículo esquerdo, hipertrofia ventricular esquerda excêntrica, cardiomiopatia hipertrófica, hipertrofia ventricular direita e 
bloqueio de ramo direito e/ou esquerdo. A DI, segundo os autores, deve ser utilizada para facilitar o diagnóstico e, em algumas circunstâncias, determinar o prognóstico, sendo considerado $45 \mathrm{~ms}$ nas derivações V5 e V6 o limite superior do normal (Pérez-Riera et al., 2016).

Este trabalho é um estudo retrospectivo, cujo objetivo foi comparar dados coletados anteriormente sobre a condição clínica e as medidas do ecocardiograma de cães diagnosticados com endocardiose de mitral e os valores da DI mensurados em seus respectivos ECGs, a fim de buscar uma relação que possa antever o diagnóstico da degeneração valvar, facilitar a classificação e fornecer o prognóstico da doença.

\section{MATERIAL E MÉTODOS}

Foram utilizados dados prévios de 45 cães, atendidos no Hospital Veterinário da Universidade Federal do Paraná - campus Curitiba, entre 2014 e 2017. Os animais passaram por avaliação clínica, eletrocardiográfica e ecocardiográfica no local; com exceção do grupo controle, todos foram diagnosticados com DMVM e classificados de acordo com o consenso ACVIM (Atkins et al., 2009). Para o presente estudo, foram divididos nos seguintes grupos: controle (10 animais), B1 (10 animais), B2 (10 animais), C (10 animais) e $\mathrm{D}$ (cinco animais). Os cães foram selecionados aleatoriamente, independentemente de sexo, raça ou idade (um a 18 anos).

Os animais passaram por exame ecocardiográfico após indicação clínica, por suspeita de cardiopatia, avaliação pré-anestésica ou check-up. O aparelho utilizado foi o Esaote MyLab30, conduzido pela equipe de Serviço de Cardiologia Veterinária do HV-UFPR. Os cães foram examinados em decúbito lateral direito e esquerdo, sem nenhum tipo de sedação. Parâmetros ecocardiográficos mensurados: átrio esquerdo (AE), aorta (Ao), relação átrio esquerdo/aorta (AE/Ao), septo interventricular na diástole (SIVd), ventrículo esquerdo na diástole (VEd), parede lateral do ventrículo esquerdo na diástole (PLVEd), septo interventricular na sístole (SIVs), ventrículo esquerdo na sístole (VEs), parede lateral do ventrículo esquerdo na sístole (PLVEs), fração de ejeção (FE), fração de encurtamento (FEC), onda $\mathrm{E}$ mitral (E mitral), onda A mitral (A mitral), relação onda E/onda $\mathrm{A}$ (E/A), tempo de relaxamento isovolumétrico (TRIV) e relação onda E/tempo de relaxamento isovolumétrico (E/TRIV).

Os pacientes foram também submetidos ao eletrocardiograma, nas derivações D1, D2, D3, aVR, aVL, aVF, rV, V2, V4 e V10. Os cães foram mantidos em decúbito lateral direito, sem sedação, e os exames performados pela mesma equipe, no aparelho ECGPC TEB. O parâmetro do ECG utilizado para correlação com a classificação clínica e os dados ecocardiográficos foi a DI, mensurada do início do complexo QRS até o ápice da onda $\mathrm{R}$, nas 10 derivações.

Dados coletados foram inseridos em planilhas Excel. A análise estatística foi feita por meio do software Minitab, na qual o teste de normalidade Anderson Darling constatou curva normal em todos os dados coletados. Em seguida, no teste paramétrico com os grupos clínicos, foi utilizado o teste de Bartlett para análise de variâncias e, ao se comprovarem variâncias estatisticamente iguais, teste ANOVA, com nível de confiança de 95\%. Valores de $\mathrm{P}<0,05$ foram considerados significativos. Por fim, realizou-se o teste de comparações múltiplas de Fisher, para determinar diferenças estatísticas entre os grupos. Os resultados com relevância estatística foram reclassificados em diferentes subgrupos, a partir do valor médio de DI do grupo clínico. Essa reclassificação foi feita para demonstrar a diferença na DI entre animais subclínicos e sintomáticos.

\section{RESULTADOS}

Os dados da análise mostraram uma diferença estatisticamente relevante nas derivações D2, $\mathrm{D} 3, \mathrm{aVF}, \mathrm{rV}, \mathrm{V} 2$ e V4 entre os grupos B1 e C e entre B2 e C. O resultado da derivação D2 está na Tab. 1. Valores médios de DI mostraram significância estatística entre o grupo $\mathrm{C}$ e os grupos $\mathrm{B} 1, \mathrm{~B} 2$ e controle $(\mathrm{P}=0,006)$, os quais foram reclassificados em subgrupos diferentes. $\mathrm{O}$ grupo D não apresentou valor médio significativamente relevante para reclassificação em apenas um subgrupo. $\mathrm{O}$ resultado da derivação D3 é mostrado na Tab. 2; nota-se o mesmo padrão de comportamento que a análise da derivação D2. 
Tabela 1. Grupo clínico da DMVM, valores médios da DI e subgrupos estatísticos na derivação D2 de 45 cães atendidos no HV-UFPR - campus Curitiba, entre 2014 e 2017

\begin{tabular}{lllll}
\hline Grupo clínico & $\mathrm{n}$ & Valor médio DI (s) & Subgrupo & \\
\hline C & 10 & 0,042900 & A & \\
D & 5 & 0,039000 & A & B \\
Controle & 10 & 0,036700 & & B \\
B2 & 10 & 0,036000 & & B \\
B1 & 10 & 0,034600 & B \\
\hline
\end{tabular}

Tabela 2. Grupo clínico da DMVM, valores médios da DI e subgrupos estatísticos na derivação D3 de 45 cães atendidos no HV-UFPR - campus Curitiba, entre 2014 e 2017

\begin{tabular}{lllll}
\hline Grupo clínico & $\mathrm{n}$ & Valor médio DI (s) & Subgrupo & \\
\hline C & 10 & 0,043300 & A & \\
D & 5 & 0,039000 & A & B \\
Controle & 10 & 0,036700 & & B \\
B2 & 10 & 0,036700 & & B \\
B1 & 10 & 0,035000 & B \\
\hline
\end{tabular}

Os valores da derivação aVF estão na Tab. 3, na qual se observa uma mudança no comportamento dos grupos. A diferença estatística entre o grupo $\mathrm{C}$ e os grupos B1 e B2 $(\mathrm{P}=0,046)$ foi mantida, porém o grupo controle apresentou o mesmo comportamento do grupo D: valor médio de DI sem relevância suficiente para ser enquadrado em apenas um subgrupo. $\mathrm{O}$ resultado da derivação rV está na Tab. 4. Nessa análise, foi observada a formação de três subagrupamentos, nos quais há diferença significativa entre o grupo $\mathrm{C}$ e o grupo $\mathrm{B} 1 \quad \mathrm{P}=0,01)$, que permanecem isolados dos demais. O grupo B2 mostrou-se diferente estatisticamente do grupo $\mathrm{C}$, porém seu comportamento passou a ser ambíguo, reclassificado em dois subgrupos. $\mathrm{O}$ grupo controle e o grupo D tiveram resultados variados. Valores da derivação V2 estão na Tab. 5, que seguiu o mesmo padrão encontrado na derivação $\mathrm{rV}$.

Por fim, dados da última derivação com significância estatística, V4, estão na Tab. 6. Nessa derivação, os grupos D, C e B2 ficaram dispostos de modo isolado, enquanto os grupos controle e B1 tiveram comportamento variável. Esse resultado foi o único em que B1 apresentouse diferente do esperado e com valor médio maior que B2.

Tabela 3. Grupo clínico da DMVM, valores médios da DI e subgrupos estatísticos na derivação aVF de 45 cães atendidos no HV-UFPR - campus Curitiba, entre 2014 e 2017

\begin{tabular}{lllll}
\hline Grupo clínico & $\mathrm{n}$ & Valor médio DI (s) & Subgrupo & \\
\hline C & 10 & 0,040900 & A & \\
D & 5 & 0,039000 & A & B \\
Controle & 10 & 0,036700 & A & B \\
B2 & 10 & 0,035600 & & B \\
B1 & 10 & 0,034600 & & B \\
\hline
\end{tabular}

Tabela 4. Grupo clínico da DMVM, valores médios da DI e subgrupos estatísticos na derivação rV de 45 cães atendidos no HV-UFPR - campus Curitiba, entre 2014 e 2017

\begin{tabular}{|c|c|c|c|c|c|}
\hline Grupo clínico & $\mathrm{n}$ & Valor médio DI (s) & \multicolumn{3}{|c|}{ Subgrupo } \\
\hline $\mathrm{C}$ & 10 & 0,036600 & $\mathrm{~A}$ & & \\
\hline $\mathrm{D}$ & 5 & 0,034600 & A & B & \\
\hline Controle & 10 & 0,032400 & $\mathrm{~A}$ & B & $\mathrm{C}$ \\
\hline B2 & 10 & 0,028800 & & B & $\mathrm{C}$ \\
\hline B1 & 10 & 0,027600 & & & $\mathrm{C}$ \\
\hline
\end{tabular}


Tabela 5. Grupo clínico da DMVM, valores médios da DI e subgrupos estatísticos na derivação V2 de 45 cães atendidos no HV-UFPR - campus Curitiba, entre 2014 e 2017

\begin{tabular}{llllll}
\hline Grupo clínico & $\mathrm{n}$ & Valor médio DI (s) & Subgrupo & & \\
\hline C & 10 & 0,036600 & A & & \\
D & 5 & 0,034600 & A & B & \\
Controle & 10 & 0,032400 & A & B & C \\
B2 & 10 & 0,028800 & & B & C \\
B1 & 10 & 0,027600 & & C \\
\hline
\end{tabular}

Tabela 6. Grupo clínico da DMVM, valores médios da DI e subgrupos estatísticos na derivação V4 de 45 cães atendidos no HV-UFPR - campus Curitiba, entre 2014 e 2017

\begin{tabular}{|c|c|c|c|c|c|}
\hline Grupo clínico & $\mathrm{n}$ & Valor médio DI (s) & Subgrupo & & \\
\hline $\mathrm{C}$ & 10 & 0,040200 & $\mathrm{~A}$ & & \\
\hline $\mathrm{D}$ & 5 & 0,039000 & $\mathrm{~A}$ & & \\
\hline Controle & 10 & 0,036200 & A & B & \\
\hline B1 & 10 & 0,032300 & & B & $\mathrm{C}$ \\
\hline $\mathrm{B} 2$ & 10 & 0,032000 & & & $\mathrm{C}$ \\
\hline
\end{tabular}

Valores médios da DI nas 10 derivações com desvio-padrão e respectivo valor de significância estatística estão na Tab. 7. O valor de $\mathrm{p}(\mathrm{P}<0,05)$ demonstrado nas derivações D2, D3, aVF, rV, V2 e V4 indica significância estatística relevante, pois animais sintomáticos (grupo C) apresentaram valores de DI maiores nessas derivações do que os animais em estágios subclínicos (B1 e B2).

Tabela 7. Valores médios da DI (s) em 10 derivações do ECG com desvio-padrão e valor p dos grupos clínicos da DMVM de 45 cães atendidos no HV-UFPR - campus Curitiba, entre 2014 e 2017

\begin{tabular}{lllllll}
\hline & Controle & B1 & B2 & C & D & Valor p \\
\hline D1 & $0,0371 \pm 0,002558$ & $0,0370 \pm 0,004000$ & $0,0360 \pm 0,004163$ & $0,0373 \pm 0,007072$ & $0,0390 \pm 0,005477$ & $0,856 *$ \\
D2 & $0,0367 \pm 0,002869$ & $0,0346 \pm 0,004719$ & $0,0360 \pm 0,004163$ & $0,0429 \pm 0,006806$ & $0,0390 \pm 0,005477$ & 0,006 \\
D3 & $0,0367 \pm 0,002869$ & $0,0350 \pm 0,005598$ & $0,0367 \pm 0,003592$ & $0,0433 \pm 0,006617$ & $0,0390 \pm 0,005477$ & 0,007 \\
AVR & $0,0367 \pm 0,002869$ & $0,0346 \pm 0,004719$ & $0,0356 \pm 0,004248$ & $0,0388 \pm 0,005534$ & $0,0390 \pm 0,005477$ & $0,214 *$ \\
AVL & $0,0370 \pm 0,0040$ & $0,0326 \pm 0,006552$ & $0,0351 \pm 0,005425$ & $0,0370 \pm 0,007318$ & $0,0346 \pm 0,005899$ & $0,449 *$ \\
AVF & $0,0367 \pm 0,002869$ & $0,0346 \pm 0,004719$ & $0,0356 \pm 0,004248$ & $0,0409 \pm 0,006437$ & $0,0390 \pm 0,005477$ & 0,046 \\
RV & $0,0317 \pm 0,005122$ & $0,0253 \pm 0,005794$ & $0,0252 \pm 0,003967$ & $0,0336 \pm 0,008695$ & $0,0278 \pm 0,005975$ & 0,01 \\
V2 & $0,0324 \pm 0,004427$ & $0,0276 \pm 0,005522$ & $0,0288 \pm 0,005350$ & $0,0366 \pm 0,006963$ & $0,0346 \pm 0,006731$ & 0,007 \\
V4 & $0,0362 \pm 0,002741$ & $0,0323 \pm 0,005034$ & $0,0320 \pm 0,003528$ & $0,0402 \pm 0,006070$ & $0,0390 \pm 0,005477$ & 0,001 \\
V10 & $0,0361 \pm 0,003178$ & $0,0353 \pm 0,004968$ & $0,0376 \pm 0,006096$ & $0,0403 \pm 0,005599$ & $0,0378 \pm 0,007430$ & $0,297 *$ \\
\hline
\end{tabular}

*P>0,05 sem significância estatística.

\section{DISCUSSÃO}

Houve variação considerável nas medidas de cada grupo, principalmente entre os grupos B1, B2 e C. Os valores encontrados mostraram-se gradativamente maiores à medida que a doença $\mathrm{e}$ o estágio clínico avançavam. No grupo C, que já apresenta sinais de insuficiência, estão os valores mais altos de DI, principalmente na derivação D2, a mais usada na avaliação eletrocardiográfica. Esses dados indicam prolongamento do QRS e despolarização
Foram relacionados os valores de DI das derivações com significado estatístico e parâmetros do ecocardiograma, porém nenhuma relação linear entre eles foi encontrada. As derivações D1, aVR, aVL, V10 tiveram P>0,05, portanto sem relevância estatística. ventricular esquerda mais lenta em relação aos grupos com DI menor. Estudos da cardiologia médica já demonstraram o impacto desse achado em pacientes humanos.

Pessoas com tempo DI > 40ms são mais propensas a ter cardiomiopatia isquêmica, revascularização do miocárdio e fibrilação atrial crônica (Vegh et al., 2017). Outros autores apontam $45 \mathrm{~ms}$ como o valor de DI no qual o risco de desenvolvimento de IC aumenta consideravelmente; isso porque a DI prolongada 
ocorre antes de alterações maiores, como o bloqueio de ramo, no desenvolvimento da IC, além de ser um fator prognóstico adicional à despolarização total, medida pela duração do QRS (O’neal et al., 2016).

No estudo de Pérez-Riera et al. (2016), o mesmo valor de $45 \mathrm{~ms}$ é mostrado como o limite superior normal em humanos, e valores acima disso podem ocorrer em diversas situações, sendo a principal delas a sobrecarga de volume e a hipertrofia ventricular esquerda, a qual é observada na regurgitação mitral. $\mathrm{O}$ intervalo de tempo de DI de 50ms nas derivações V5 e V6 é um critério para hipertrofia ventricular esquerda devido à sobrecarga e à disfunção diastólica (Pérez-Riera et al., 2016). Valores ainda maiores (DI $>60 \mathrm{~ms}$ ) já foram mensurados em pessoas e associados a casos de bloqueio de ramo esquerdo e atraso de condução intraventricular esquerda não específico; a DI foi usada ainda como preditor de resposta à terapia de ressincronização cardíaca (TRC) em pacientes com IC, fração de ejeção do VE baixa e QRS prolongado (Pastore et al., 2016).

A terapia de ressincronização cardíaca é uma opção terapêutica eficaz para a IC congestiva avançada e refratária a fármacos (Del Carpio et al., 2013) e já havia sido estudada anteriormente, seguindo os mesmos critérios de inclusão de pacientes: presença de IC, baixa fração de ejeção do VE e duração prolongada do QRS. O tempo de DI mostrou-se um melhor preditor da resposta ao tratamento quando comparado à duração do QRS (Del Carpio et al., 2013), o que poderia auxiliar na condução do tratamento de cães com IC refratária.

Além dos benefícios já mostrados no uso da DI para complementar o diagnóstico, monitorar o tratamento e estimar prognóstico dos pacientes humanos, principalmente nos casos de hipertrofia ventricular esquerda, um estudo quantificou a relação direta da medida eletrocardiográfica com parada cardíaca. Uma DI prolongada, > 0,05s (V5 e V6), estaria associada a aumento da massa e disfunção do VE, mas está relacionada principalmente com risco maior de morte súbita independentemente da hipertrofia ventricular esquerda, diagnosticada tanto pelo ecocardiograma quanto pelo ECG, e da fração de ejeção ventricular esquerda reduzida, sugerindo que o valor prognóstico dessa medida vai além das modificações no ventrículo esquerdo, pois reflete um remodelamento elétrico anormal no miocárdio como um todo (Darouian et al., 2016).

O grupo controle mostrou um comportamento diferente do esperado para animais sem DMVM, principalmente nas últimas derivações, por não apresentar valores de DI mais baixos do que os diagnosticados. Em todas as derivações, a DI média do grupo controle foi igual ou maior do que a DI média dos grupos B1 e B2. Tal efeito poderia ser explicado pela ampla faixa etária dos animais do grupo controle (um a 11 anos), que não apresentavam diagnóstico de DMVM, mas poderiam ter outras doenças, condições subclínicas ou fazer uso de medicações capazes de prolongar a despolarização ventricular, independentemente da condição das valvas.

Já no grupo D, o comportamento foi semelhante ao do grupo $\mathrm{C}$, valores altos em todas as derivações; entretanto, sendo o grupo D o último grupo de classificação clínica da DMVM, o grupo que abrange pacientes terminais $\mathrm{e}$ refratários à terapia convencional, seria esperado que esses animais apresentassem valores ainda maiores que no grupo $\mathrm{C}$, compatíveis com prognóstico reservado a ruim. Resultados inesperados como esse podem ter ocorrido em razão do $\mathrm{n}$ menor do grupo $\mathrm{D}$ (cinco animais) e pelo fato de as modificações estruturais $\mathrm{e}$ funcionais nas câmaras gerarem alterações eletrocardiográficas não diretamente relacionadas à deflexão intrinsecoide e despolarização ventricular deficiente.

Novos estudos são necessários para correlacionar DI, sobrecarga de volume e hipertrofia ventricular esquerda com um número maior de animais e critérios mais delimitados de inclusão na pesquisa, bem como um grupo controle composto por animais jovens e livres de quaisquer condições clínicas associadas a desequilíbrio hidroeletrolítico.

\section{CONCLUSÃO}

O presente estudo concluiu que pacientes de DMVM apresentam um aumento gradativo no valor da DI à medida que a doença avança, principalmente nos estágios B1, B2 e C. Cães em estágios avançados apresentam a DI mais prolongada do que pacientes em estágios mais brandos, o que indica uma despolarização 
ventricular mais lenta possivelmente associada à sobrecarga e à hipertrofia do ventrículo esquerdo. Exames para avaliar distúrbios elétricos no ciclo cardíaco vêm aprimorando a eletrofisiologia e a detecção de alterações discretas em enfermidades subclínicas. Sendo a DI uma medida mais precisa da despolarização ventricular, ela pode ser usada como parâmetro de classificação de cães acometidos pela DMVM.

\section{REFERÊNCIAS}

ATKINS, C.; BONAGURA, J.; ETTINGER, S. et al. Guidelines for the diagnosis and treatment of canine chronic valvular heart disease. J. Vet. Intern. Med., v.23, p.1142-1150, 2009.

BORGARELLI, M.; BUCHANAN, J.W. Historical review, epidemiology and natural history of degenerative mitral valve disease. $J$. Vet. Cardiol., v.14, p.93-101, 2012.

CHETBOUL, V.; TISSIER, R. Echocardiographic assessment of canine degenerative mitral valve disease. J. Vet. Cardiol., v.14, p.127-148, 2012.

CROSARA, S.; BORGARELLI, M.; PEREGO, M. et al. Holter monitoring in 36 dogs with myxomatous mitral valve disease. Aust. Vet. J., v.88, p.386-392, 2010.

DAROUIAN, N.; NARAYANAN, K.; ARO, A.L. et al. Delayed intrinsicoid deflection of the QRS complex is associated with sudden cardiac arrest. Heart Rhythm, v.13, p.927-932, 2016.

DEL CARPIO, M.F.; POWELL, B.D.; CHA, Y.M. et al. Delayed intrinsicoid deflection onset in surface ECG lateral leads predicts left ventricular reverse remodeling after cardiac resynchronization therapy. Heart Rhythm, v.10, p.979-987, 2013.

HÄGGSTRÖM, J.; PEDERSEN, H.D.; KVART, C. New insights into degenerative mitral valve disease in dogs. Vet. Clin. Small Anim., v.34, p.1209-1226, 2004.

O’NEAL, W.T.; QURESHI, W.T.; NAZARIAN, S. et al. Electrocardiographic time to intrinsicoid deflection and heart failure: the multi-ethnic study of atherosclerosis. Clin. Cardiol., v.39, p.531-536, 2016.
OLIVEIRA, M.S.; MUZZI, R.A.L.; ARAÚJO, R.B. et al. Heart rate variability and arrhythmias evaluated with Holter in dogs with degenerative mitral valve disease. Arq. Bras. Med. Vet. Zootec., v.66, p.425-432, 2014.

PARKER, H.G.; KILROY-GLYNN, P. Myxomatous mitral valve disease in dogs: does size matter? J. Vet. Cardiol., v.14, p.19-29, 2012.

PASTORE, G.; MAINES, M.; MARCANTONI, L. et al. ECG parameters predict left ventricular conduction delay in patients with left ventricular dysfunction. Heart Rhythm, v.13, p.2289-2296, 2016.

PÉREZ-RIERA, A.R.; ABREU, L.C.; BARBOSA-BARROS, R. et al. R-Peak time: an electrocardiographic parameter with multiple clinical applications. Ann. Noninvasive Electrocardiol., v.21, p.10-19, 2016.

PETRUS, L.C.; LARSSON, M.H.M.A. Valvulopatias adquiridas. In: JERICO, M.M.; ANDRADE NETO, J.P.; KOGIKA, M.M. (Eds.). Tratado de medicina interna de cães $e$ gatos. Rio de Janeiro: Roca, 2015. p.1162-1179.

RASMUSSEN C.E.; FALK, T.; ZOIS, N.E. et al. Heart rate, heart rate variability, and arrhythmias in dogs with myxomatous mitral valve disease. J. Vet. Intern. Med., v.26, p.76-84, 2012.

ROMHILT, D.W.; ESTES, E.H.Jr. A point-score system for the ECG diagnosis of left ventricular hypertrophy. Am. Heart J., v.75, p.752-758, 1968.

TILLEY, L.P. Analysis of canine P-QRS-S deflections. In: TILLEY, L.P. (Ed.). Essentials of canine and feline electrocardiography: interpretation and treatment. Philadelphia: Lea \& Febiger, 1992. p.59-99.

VÉGH, E.M.; KANDALA, J.; JANUSZKIEWICZ, L. et al. A new simplified electrocardiographic score predicts clinical outcome in patients treated with CRT. Europace, v.0, p.1-9, 2017. 